\title{
The Topological Sensitivity with respect to Furstenberg Families
}

\author{
Tengfei Wang, Kai Jing, and Jiandong Yin $\mathbb{D}$ \\ Department of Mathematics, Nanchang University, Nanchang 330031, China \\ Correspondence should be addressed to Jiandong Yin; yjdaxf@163.com
}

Received 20 February 2020; Accepted 16 May 2020; Published 4 June 2020

Academic Editor: Cengiz Çinar

Copyright (C) 2020 Tengfei Wang et al. This is an open access article distributed under the Creative Commons Attribution License, which permits unrestricted use, distribution, and reproduction in any medium, provided the original work is properly cited.

In this work, a dynamical system $(X, f)$ means that $X$ is a topological space and $f: X \longrightarrow X$ is a continuous map. The aim of the article is to introduce the conceptions of topological sensitivity with respect to Furstenberg families, $n$-topological sensitivity, and multisensitivity and present some of their basic features and sufficient conditions for a dynamical system to possess some sensitivities. Actually, it is proved that every topologically ergodic but nonminimal system is syndetically sensitive and a weakly mixing system is $n$-thickly topologically sensitive and multisensitive under the assumption that $X$ admits some separability.

\section{Introduction}

For a compact system $(X, f)$ which means that $f$ is a continuous self-map on a compact metric space $(X, d)$, sensitive dependence on initial condition (sensitive for simplicity) for $(X, f)$ was firstly introduced by Ruelle [1] as if there exists $\delta>0$ such that for each $x \in X$ and every open neighborhood $V_{x}$ of $x$, there is a nonnegative integer $n$ such that $\sup \left\{d\left(f^{n}(x), f^{n}(y)\right): y \in V_{x}\right\}>\delta$. One can write this in a slightly different way (see [2]) as follows. For a nonempty $V \subset X$ and $\delta>0$, let

$$
S_{f}(V, \delta)=\left\{n \in \mathbb{N}_{0}: \exists x, y \in V \text {, s.t.d }\left(f^{n}(x), f^{n}(y)\right)>\delta\right\},
$$

where $\mathbb{N}_{0}$ denotes the set of nonnegative integers. Then, a compact system $(X, f)$ is sensitive if and only if there exists $\delta>0$ such that $S_{f}(V, \delta) \neq \varnothing$ for each nonempty open (opene for simplicity) subset $V$ of $X$.

Sensitivity is a key conception used to characterize the unpredictability of a compact system and a chief component of some chaotic properties such as the chaos in the sense of Devaney [3] and Banks et al. [4]. In [5, 6], the authors introduced the linear chaos and linear topological dynamics, and one can in [7] for the concept of multivalued linear and nonlinear topological dynamics. More detailed information of the related studies of sensitivity are introduced in [2, 8-13] and [14]. For the sake of distinguishing the following topological version of sensitivity, we use the classical sensitivity to stand for the sensitivity of compact systems in this paper.

From now on, we call the pair $(X, f)$ a dynamical system if $f$ is a continuous self-map on a topological space $X$. In [15], the author introduced the topological version of sensitivity (topological sensitivity for short) for dynamical systems as follows.

Definition 1 (see [15]). Let $(X, f)$ be a dynamical system. An open cover $\mathcal{U}$ of $X$ is called a sensitivity cover (s-cover for short) for $(X, f)$ if for every opene subset $G$ of $X$ there exist $x, y \in G$ and $n \in \mathbb{N}_{0}$ such that $(x, y) \notin \cup\left\{f^{-n}(U) \times f^{-n}(U): U \in \mathcal{U}\right\}$.

Definition 2 (see [15]). A dynamical system $(X, f)$ (or simply the map $f)$ is called topologically sensitive if $(X, f)$ has an $s$-cover.

In other words, a dynamical system is topologically sensitive if it admits an open cover $\mathcal{U}$ satisfying that, for every opene set $G$ in $X$, there are $x, y \in G$ and $n \in \mathbb{N}_{0}$ such that $(x, y) \notin f^{-n}(U) \times f^{-n}(U)$ for each $U \in \mathcal{U}$.

Topological sensitivity of dynamical systems generalizes the classical sensitivity of compact systems since there exist in [5] dynamical systems on metric noncompact spaces which are topologically sensitive but not classically sensitive. The author 
of [15] presented some sufficient conditions for a dynamical system to be topologically sensitive. For example, it was proven in [15] that each transitive map possessing a dense set of almost periodic points and admitting an eventually periodic point on an infinite Urysohn space is topologically sensitive; every weakly mixing map is topologically sensitive if there are two opene subsets in the phase space such that the intersection of their closures is empty. Moreover, the weakly positively expansive maps were also considered in [15].

In this paper, for the sake of dealing with the conception of topological sensitivity of dynamical systems in a unified way, we introduce the conceptions of topological sensitivity with respect to families of $\mathbb{N}_{0}, n$-topological sensitivity, and multisensitivity for dynamical systems and give some sufficient conditions for a dynamical system to possess distinct sensitivities. Some of the presented results improve or generalize the main results of [15] to a great extent.

\section{Preliminaries}

In this section, we recall some notations, notions and basic theories of nonnegative integers and dynamical systems.

2.1. Subsets of Nonnegative Integers. Throughout this paper, denote by $\mathbb{N}_{0}$ the set of nonnegative integers, $\mathbb{N}$ the set of positive integers, $\mathbb{Z}$ the set of integers, and $\mathbb{R}$ the set of real numbers, respectively.

Let $\mathscr{P}$ be the collection of all subsets of $\mathbb{N}_{0}$. A subset $\mathscr{F}$ of $\mathscr{P}$ is called a Furstenberg family (family for short) of $\mathbb{N}_{0}$ provided it is hereditary upward, that is, $F_{1} \subset F_{2}$ and $F_{1} \in \mathscr{F}$ imply $F_{2} \in \mathscr{F}$. A family $\mathscr{F}$ of $\mathbb{N}_{0}$ is proper if it is a proper subset of $\mathscr{P}$, namely, $\mathscr{F} \neq \varnothing$ and $\mathscr{F} \neq \mathscr{P}$; translation invariant if for each $n \in \mathbb{N}_{0}, F+n=\{i+n: i \in F\} \in \mathscr{F}$ for each $F \in \mathscr{F}$; and a filter if it is proper and closed under intersection, i.e., $F_{0}, F_{1} \in \mathscr{F}$ implies $F_{0} \cap F_{1} \in \mathscr{F}$.

Let $\mathscr{F}$ be a family of $\mathbb{N}_{0}$, write $[\mathscr{F}]=\{A \in \mathscr{P}$ : there exists $F \in \mathscr{F}$ such that $F \subset A\}$,

namely, $[\mathscr{F}]$ is the smallest family generated by the family $\mathscr{F}$.

A set $F \subset \mathbb{N}_{0}$ is called thick if for each $m \in \mathbb{N}$ there exists $t_{m} \in \mathbb{N}_{0}$ such that $\left\{t_{m}, t_{m}+1, \ldots, t_{m}+m\right\} \subset F$ and syndetic if there exists $m \in \mathbb{N}$ such that, for every $t \in \mathbb{N}_{0}$, $\{t, t+1, \ldots, t+m\} \cap F \neq \varnothing$. By their definitions, it is obvious that every thick subset of $\mathbb{N}_{0}$ intersects each syndetic subset of $\mathbb{N}_{0}$.

A set $F \subset \mathbb{N}_{0}$ is called piecewise syndetic if it can be written as the intersection of a thick set and a syndetic set and thickly syndetic if for any $n \in \mathbb{N}$, there exists a syndetic set $\left\{s_{1}^{n}<s_{2}^{n}<\cdots\right\}$ such that

$$
\bigcup_{j=1}^{\infty}\left\{s_{j}^{n}, s_{j}^{n}+1, \ldots, s_{j}^{n}+n\right\} \subset F .
$$

The upper density of a subset $S$ of $\mathbb{N}_{0}$ is defined as

$$
\limsup _{n \longrightarrow \infty} \frac{1}{n}|S \cap\{0,1,2, \ldots, n-1\}|,
$$

where $|A|$ denotes the cardinality of the set $A$. Similarly one can define the lower density of $S \subset \mathbb{N}_{0}$.
The upper Banach density of $S \subset \mathbb{N}_{0}$ is defined as

$$
\underset{|I| \longrightarrow \infty}{\limsup } \frac{|S \cap I|}{|I|} \text {. }
$$

The supremun is taken over all segments of $\mathbb{N}_{0}$. One can see [16] for more details of families.

In general, we use $\mathscr{F}_{\text {inf }}$ to denote the family consisting of all infinite subsets of $\mathbb{N}_{0}$ and use $\mathscr{F}_{\mathrm{s}}, \mathscr{F}_{\mathrm{t}}, \mathscr{F}_{\text {ts }}, \mathscr{F}_{\mathrm{ps}}, \mathscr{F}_{\text {pud }}$, $\mathscr{F}_{\text {pld }}$, and $\mathscr{F}_{\text {pubd }}$ to denote, respectively, the families of syndetic subsets, thick subsets, thickly syndetic subsets, piecewise syndetic subsets, the subsets with positive upper density, the subsets with positive lower density, and the subsets with positive upper Banach density of $\mathbb{N}_{0}$. Then, $\mathscr{F}_{s}$, $\mathscr{F}_{\text {t }}, \mathscr{F}_{\text {ts }}, \mathscr{F}_{\text {ps }}, \mathscr{F}_{\text {pud }}, \mathscr{F}_{\text {pld }}$, and $\mathscr{F}_{\text {pubd }}$ are proper and translation invariant. About the sets with positive upper Banach density, there is a result in [16] stated as follows which will be used in the proof of Lemma 2 in the paper.

Proposition 1 (see [16]). For each $S \in \mathscr{F}_{\text {pubd }}, S-S$ is syndetic. Here, $S-S=\{i-j \geq 0: i, j \in S\}$.

2.2. Basic Notions of Dynamical Systems. Let $(X, f)$ be a dynamical system. Let $\bar{A}$ denote the closure of $A \subset X$ in $X$ and $A-B$ denotes the difference set of $A, B$, i.e., $A-B=\{a: a \in A, a \notin B\}$.

For nonempty open (opene in brief and hereinafter) subsets $U, V$ of $X$ and $x \in X$, let $\mathcal{U}_{x}$ denote the collection of all open neighborhoods of $x$ and write $N(U, V)=\left\{n \in \mathbb{N}_{0}: U \cap f^{-n}(V) \neq \varnothing\right\} \quad$ and $\quad N(x, U)=$ $\left\{n \in \mathbb{N}_{0}: f^{n}(x) \in U\right\}$.

A point $x \in X$ is a transitive point of $f$ if $\operatorname{orb}(x)$, the orbit of $x$ under $f$, is dense in $X$. A dynamical system $(X, f)$ is minimal if each point in $X$ is transitive. In a dynamical system $(X, f), x \in X$ is called a minimal point if the dynamical system $(\overline{\operatorname{orb}(x)}, f \mid \overline{\operatorname{orb}(x)})$ is minimal. A subset $M$ of $X$ is called minimal if every point of $M$ is minimal.

A point $x \in X$ is called an almost periodic point of $f$ if $N(x, U) \in \mathscr{F}_{s}$ for each $U \in \mathscr{U}_{x}$. Denote by $A(f)$ the set of all almost periodic points of $f$. In [17], the authors introduced the concept of positive upper Banach density recurrent points for compact systems. Now, we introduce this notion for dynamical systems.

Definition 3. Let $(X, f)$ be a dynamical system. A point $x \in X$ is called a positive upper Banach density recurrent point of $f$ if $N(x, U) \in \mathscr{F}_{\text {pubd }}$ for each $U \in \mathscr{U}_{x}$.

Denote by $B D^{*}(f)$ the set of all positive upper Banach density recurrent points of $f$. Obviously, $A(f) \subset B D^{*}(f)$.

Let $\mathscr{F}$ be a family of $\mathbb{N}_{0}$. A dynamical system $(X, f)$ is $\mathscr{F}$-transitive if for each pair of opene subsets $U, V$ of $X$, $N(U, V) \in \mathscr{F} ; \mathscr{F}$-central if for each opene subset $V$ of $X$, $N(V, V) \in \mathscr{F}$. Especially, a dynamical system $(X, f)$ is transitive if for each pair of opene subsets $U, V$ of $X$, $N(U, V) \neq \varnothing$; topological ergodic if $N(U, V) \in \mathscr{F}_{\text {s }}$ for each pair of opene subsets $U, V$ of $X$; weakly mixing if the product system $(X \times X, f \times f)$ is transitive. In Section 3, we will prove that a weakly mixing system possesses some analogous 
properties for the hitting time set of any two opene subsets to compact systems.

A dynamical system $(X, f)$ is called an $M$-system if it is transitive and the set of its minimal points is dense in $X$.

A dynamical system $(X, f)$ is semiconjugate to a dynamical system $(Y, g)$ if there exists a continuous surjection $\pi: X \longrightarrow Y$ such that $\pi^{\circ} f=g^{\circ} \pi$. Meanwhile, $\pi$ is called a semiconjugation from $f$ to $g$. Moreover, a semiconjugation $\pi$ from $f$ to $g$ is semiopen if $\pi(U)$ has a nonempty interior for each opene $U \subset X$. Especially, if $\pi$ is a homeomorphism from $X$ to $Y$, then $(X, f)$ is said to be conjugate to $(Y, g)$.

Now, based on Definitions 1 and 2, we introduce a more general version of topological sensitivity for a dynamical system. Actually, we introduce the notion of topological sensitivity with respect to a family of $\mathbb{N}_{0}$ stated as below.

Definition 4. Let $(X, f)$ be a dynamical system, $\mathscr{F}$ be a family of $\mathbb{N}_{0}$, and $\mathcal{U}$ be an open cover of $X$. $\mathcal{U}$ is called an $\mathscr{F}$-sensitivity cover for $(X, f)$ if for each opene subset $G$ of $X$,

$$
\begin{aligned}
N_{f}(G, \mathcal{U}):= & \left\{n \in \mathbb{N}_{0}: \exists x, y \in G \text { s.t. }(x, y)\right. \\
& \left.\notin \cup\left\{f^{-n}(U) \times f^{-n}(U): U \in \mathcal{U}\right\}\right\} \in \mathscr{F} .
\end{aligned}
$$

Now, by using the notion of $\mathscr{F}$-sensitivity covers, we introduce the topological version of sensitivity with respect to a Furstenberg family for dynamical systems.

Definition 5. Let $\mathscr{F}$ be a family of $\mathbb{N}_{0}$. A dynamical system $(X, f)$ is called $\mathscr{F}$-topologically sensitive if $(X, f)$ admits an $\mathscr{F}$-sensitivity cover.

Obviously, a dynamical system $(X, f)$ is topologically sensitive if and only if it is $\mathscr{F}$-topologically sensitive with respect to the family $\mathscr{F}=\mathscr{P}-\{\varnothing\}$ and the notion of \{family\}-topological sensitivity of dynamical systems generalizes that of $\{$ family $\}$-sensitivity of compact systems.

Example 1. Let $f$ be the self-map on $\mathbb{R}$ defined by $f(x)=x+1$, and let us consider the following metric on $\mathbb{R}$ : $\rho(x, y)=\left|e^{x}-e^{y}\right|$. Then, this metric is equivalent to the usual metric $d$ on $\mathbb{R}$, namely, they generate the same topology of $\mathbb{R}$. From [15], it is known that $(\mathbb{R}, f)$ is topologically sensitive. In fact, it is not hard to check that $(\mathbb{R}, f)$ is $\mathscr{F}$-topologically sensitive for the families $\mathscr{F}:=\mathscr{F}_{\mathrm{s}}, \mathscr{F}_{\mathrm{t}}, \mathscr{F}_{\mathrm{ts}}, \mathscr{F}_{\mathrm{ps}}, \mathscr{F}_{\text {pld }}, \mathscr{F}_{\text {pubd }}$.

Take an open cover $\mathscr{U}=\{B(p, e / 2): p \in \mathbb{R}\}$ of $\mathbb{R}$. For any opene subset $G$ of $\mathbb{R}$ and $x, y \in G$ with $x \neq y$, let $F_{x, y}=\left\{\left[\ln \left(1 /\left|e^{x}-e^{y}\right|\right)\right]+2+i: i \in \mathbb{N}_{0}\right\}$. Obviously, each $F_{x, y}$ is cofinite, i.e., $\mathbb{R}-F_{x, y}$ is finite. Put $\mathscr{F}=\left\{F_{x, y}: x, y \in G, x \neq y, G \subset \mathbb{R}\right.$ is nonempty and open $\}$.

For any opene subset $G$ of $\mathbb{R}$ and $x, y \in G$ with $x \neq y$ and $n_{i}:=\left[\ln \left(1 /\left|e^{x}-e^{y}\right|\right)\right]+2+i \in F_{x, y}$, we have

$$
(x, y) \notin \cup\left\{f^{-n_{i}}(U) \times f^{-n_{i}}(U): U \in \mathcal{U}\right\} .
$$

That is, $\left\{f^{n_{i}}(x), f^{n_{i}}(y)\right\} \nsubseteq B(p, e / 2)$ for each $p \in \mathbb{R}$ and $i \in \mathbb{N}_{0}$. If not, there exist some opene subset $G$ of $\mathbb{R}$ and $x, y \in G$ with $x \neq y$ and $q \in \mathbb{R}$ and $j \in \mathbb{N}_{0}$ such that $\rho\left(f^{n_{j}}(x), q\right)<(e / 2)$ and $\rho\left(f^{n_{j}}(y), q\right)<(e / 2)$; then,

$$
\rho\left(f^{n_{j}}(x), f^{n_{j}}(y)\right) \leq \rho\left(f^{n_{j}}(x), q\right)+\rho\left(f^{n_{j}}(y), q\right)<\frac{e}{2}+\frac{e}{2}=e .
$$

On the contrary,

$$
\begin{aligned}
\rho\left(f^{n_{j}}(x), f^{n_{j}}(y)\right)= & \left|e^{x+n_{j}}-e^{y+n_{j}}\right|=e^{\left[\ln \left(1 /\left|e^{x}-e^{y}\right|\right)\right]+2+j} \\
& \cdot\left|e^{x}-e^{y}\right|>e^{1+j} \geq e,
\end{aligned}
$$

which is a contradiction. Therefore, for every opene set $G \subset \mathbb{R}$ and every pair $(x, y) \in G \times G$ with $x \neq y$, $F_{x, y} \subset N_{f}(G, \mathscr{U})$ and $N_{f}(G, \mathscr{U}) \in \mathscr{F}$. It implies that $\mathscr{U}=$ $\{B(p,(e / 2)): p \in \mathbb{R}\}$ is an $\mathscr{F}$-sensitivity cover of $f$.

\section{Topological Sensitivity with respect to Families of Dynamical Systems}

In this section, we give some basic properties of $\mathscr{F}$-topological sensitivity and prove that the $\mathscr{F}$-topological sensitivity is invariant under semiconjugations and that a dynamical system $(X, f)$ is $\mathscr{F}_{\mathrm{s}}$-topologically sensitive (resp. $\mathscr{F}_{\text {t }}, \mathscr{F}_{\text {ps }}, \mathscr{F}_{\text {ts }}, \mathscr{F}_{\text {pud }}, \mathscr{F}_{\text {pld }}, \mathscr{F}_{\text {pubd }}$-topologically sensitive) if and only if so is $\left(X, f^{n}\right)$ for each $n \in \mathbb{N}$ if and only if so is $\left(X, f^{n}\right)$ for some $n \in \mathbb{N}$.

Theorem 1. Suppose that $(X, f)$ and $(Y, g)$ are two $d y$ namical systems and $\mathscr{F}$ is a family of $\mathbb{N}_{0}$. If $(X, f)$ and $(Y, g)$ are semiconjugate and the semiconjugation $\varphi$ from $f$ to $g$ is semiopen and $(Y, g)$ is $\mathscr{F}$-topologically sensitive, then so is $(X, f)$.

Proof. Let $\mathscr{V}$ be an $\mathscr{F}$-sensitivity cover for $(Y, g)$. Set $\mathcal{U}=\left\{\varphi^{-1}(V): V \in \mathscr{V}\right\}$, then $\mathcal{U}$ is an open cover of $X$. For each opene subset $G$ of $X, \varphi(G)$ has a nonempty interior since $\varphi$ is semiopen. Take an opene set $W \subset \varphi(G)$. By the $\mathscr{F}$-topological sensitivity of $(Y, g)$,

$$
\begin{aligned}
N_{g}(W, \mathscr{V})= & \left\{n \in \mathbb{N}_{0}: \exists x, y \in W \text { s.t. }(x, y) \notin\right. \\
& \left.\cup\left\{g^{-n}(V) \times g^{-n}(V): V \in \mathscr{V}\right\}\right\} \in \mathscr{F} .
\end{aligned}
$$

Thus, for each $n \in N_{g}(W, \mathscr{V})$, there exist $y_{n}, \widetilde{y}_{n} \in W$ such that, for every $V \in \mathscr{V}, y_{n} \notin g^{-n}(V)$ or $\tilde{y}_{n} \notin g^{-n}(V)$. Take $x_{n}, \widetilde{x}_{n} \in G$ such that $y_{n}=\varphi\left(x_{n}\right)$ and $\tilde{y}_{n}=\varphi\left(\tilde{x}_{n}\right)$. Then, for every $U \in \mathcal{U}, \quad x_{n} \notin f^{-n}(U)$ or $\tilde{x}_{n} \notin f^{-n}(U)$. So, $N_{g}(W, \mathscr{V}) \subset N_{f}(G, \mathscr{U})$. The definition of families implies $N_{f}(G, \mathscr{U}) \in \mathscr{F}$ for each opene subset $G$ of $X$. Therefore, $(X, f)$ is $\mathscr{F}$-topologically sensitive.

In the following, we will show that the $\mathscr{F}_{\mathrm{s}}$-topological sensitivity as well as $\mathscr{F}_{\text {t }}, \mathscr{F}_{\text {ps }}, \mathscr{F}_{\text {ts }}, \mathscr{F}_{\text {pud }}, \mathscr{F}_{\text {pld }}$, and

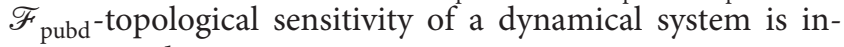
variant under iterations.

Theorem 2. Let $(X, f)$ be a dynamical system. Then, the following statements are equivalent:

(1) $(X, f)$ is $\mathscr{F}_{s}$-topologically sensitive (resp. $\mathscr{F}_{t}, \mathscr{F}_{p s}$, $\mathscr{F}_{\text {ts }}$-topologically sensitive) 
(2) $\left(X, f^{n}\right)$ is $\mathscr{F}_{s}$-topologically sensitive (resp. $\mathscr{F}_{t}, \mathscr{F}_{p s}$, $\mathscr{F}_{\text {ts }}$-topologically sensitive) for each $n \in \mathbb{N}$

(3) $\left(X, f^{n}\right)$ is $\mathscr{F}_{s}$-topologically sensitive (resp. $\mathscr{F}_{t}, \mathscr{F}_{p s}$, $\mathscr{F}_{\text {ts }}$-topologically sensitive) for some $n \in \mathbb{N}$

Proof. We only prove the case of $\mathscr{F}_{\mathrm{s}}$-topological sensitivity since the proofs of other cases are similar:

$(1) \Rightarrow(2)$ : assume that $\mathcal{U}$ is an $\mathscr{F}_{\mathrm{s}}$-sensitivity cover for $(X, f)$ and $n \in \mathbb{N}$. For every $t \in\{0, \ldots, n-1\}$, let $\mathscr{U}_{t}=\left\{f^{-t}(U): U \in \mathscr{U}\right\}$. Set $\mathscr{V}$ as the intersection of all the covers $\mathscr{U}_{0}, \ldots, \mathscr{U}_{n-1}$, i.e., $\mathscr{V}$ is the open cover of $X$ consisting of all (nonempty) sets with the form of $U_{0} \cap U_{1} \cap \cdots \cap U_{n-1}$ with $U_{t} \in \mathscr{U}_{t}$ for $t=0,1, \ldots, n-1$. Now, take arbitrarily an opene subset $G$ of $X$, then $N_{f}(G, \mathcal{U}) \in \mathscr{F}_{s}$. For each $m \in N_{f}(G, \mathcal{U})$, there exist $x_{m}, y_{m} \in G$ such that $x_{m} \notin f^{-m}(U)$ or $y_{m} \notin f^{-m}(U)$ for every $U \in \mathcal{U}$. Clearly, $m=t_{m} n+s_{m}$ for some $t_{m} \in \mathbb{N}_{0}$ and $0 \leq s_{m} \leq n-1$. Hence, $x_{m} \notin f^{-t_{m} n-s_{m}}(U)$ or $y_{m} \notin f^{-t_{m} n-s_{m}}(U)$ for every $U \in \mathcal{U}$ which implies that $x_{m} \notin\left(f^{n}\right)^{-t_{m}}(W)$ or $y_{m} \notin\left(f^{n}\right)^{-t_{m}}(W)$ for every $W \in \mathscr{W}$. Obviously, $\left\{t_{m}: m \in N_{f}(G, \mathcal{U})\right\} \in \mathscr{F}_{s}$. This turns out that $\left(X, f^{n}\right)$ is $\mathscr{F}_{\mathrm{s}}$-topologically sensitive.

$(2) \Rightarrow(3)$ is obvious.

$(3) \Rightarrow(1)$ is easy, so we leave it to the reader.

Theorem 3. Let $(X, f)$ be a dynamical system. Then, the following statements are equivalent:

(1) $(X, f)$ is $\mathscr{F}_{\text {pud }}$-topologically sensitive (resp. $\mathscr{F}_{\text {pld }}$, $\mathscr{F}_{\text {pubd-topologically sensitive) }}$

(2) $\left(X, f^{n}\right)$ is $\mathscr{F}_{\text {pud }}$-topologically sensitive (resp. $\mathscr{F}_{\text {pld }}$,

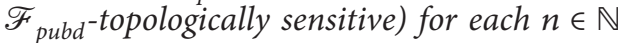

(3) $\left(X, f^{n}\right)$ is $\mathscr{F}_{\text {pud }}$-topologically sensitive (resp. $\mathscr{F}_{\text {pld }}$,

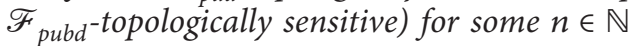

Proof. We only prove the case of $\mathscr{F}_{\text {pud }}$-topological sensitivity since the proofs of other cases are similar:

$(1) \Rightarrow(2)$ : assume that $\mathcal{U}$ is an $\mathscr{F}_{\text {pud-sensitivity cover }}$ for $(X, f)$ and $n \in \mathbb{N}$. For every $t \in\{0, \ldots, n-1\}$, let $\mathcal{U}_{t}=\left\{f^{-t}(U): U \in \mathscr{U}\right\}$. Set $\mathscr{V}$ as the intersection of all the covers $\mathscr{U}_{0}, \ldots, \mathscr{U}_{n-1}$, i.e., $\mathscr{V}$ is the open cover of $X$ consisting of all (nonempty) sets with the form of $U_{0} \cap U_{1} \cap \cdots \cap U_{n-1}$ with $U_{t} \in \mathscr{U}_{t}$ for $t=0,1, \ldots, n-1$. Now, for any opene subset $G$ of $X, N_{f}(G, \mathcal{U}) \in \mathscr{F}_{\text {pud }}$. For each $m \in N_{f}(G, \mathcal{U})$, there exist $x_{m}, y_{m} \in G$ such that $x_{m} \notin f^{-m}(U)$ or $y_{m} \notin f^{-m}(U)$ for every $U \in \mathcal{U}$. Clearly, $m=t_{m} n+s_{m}$ for some $t_{m} \in \mathbb{N}_{0}$ and $0 \leq s_{m} \leq n-1$. Hence, $x_{m} \notin f^{-t_{m} n-s_{m}}(U)$ or $y_{m} \notin f^{-t_{m} n-s_{m}}(U)$ for every $U \in \mathcal{U}$ which implies that $x_{m} \notin\left(f^{n}\right)^{-t_{m}}(W)$ or $y_{m} \notin\left(f^{n}\right)^{-t_{m}}(W)$ for every $W \in \mathscr{W}$. Then,

$$
\begin{aligned}
& \quad n \limsup _{m \rightarrow \infty} \frac{\left|N_{f^{n}}(G, \mathscr{W}) \cap\left\{0,1, \ldots, t_{m}-1\right\}\right|}{t_{m}} \\
& \quad \geq \limsup _{m \rightarrow \infty} \frac{\left|N_{f}(G, \mathscr{U}) \cap\left\{0,1, \ldots, n t_{m}+s_{m}\right\}\right|}{t_{m} n+s_{m}+1}>0 .
\end{aligned}
$$

$(2) \Rightarrow(3)$ is obvious.

$(3) \Rightarrow(1)$ is not difficult, so we leave it to the reader.

Theorem 4. If a dynamical system $(X, f)$ is topologically sensitive and there is no isolated points in $X$ and $f$ is semiopen, then $N_{f}(G, \mathcal{U})$ is infinite for each opene subset $G$ of $X$, where $\mathcal{U}$ is a sensitivity cover for $(X, f)$.

Proof. In fact, if there exists some opene set $G \subset X$ such that $N_{f}(G, \mathcal{U})$ is finite, put $n=\max \left\{k: k \in N_{f}(G, \mathcal{U})\right\}$, then $n \in N_{f}(G, \mathcal{U})$. So, there exist $x, y \in G$ such that

$$
(x, y) \notin \cup\left\{f^{-n}(U) \times f^{-n}(U): U \in \mathcal{U}\right\} .
$$

Note $f^{n+1}(x) \in X$, and there exists some $V \in \mathcal{U}$ such that $f^{n+1}(x) \in V$. Since $f^{n+1}$ is continuous, there exists $U_{x} \in \mathcal{U}_{x}$ such that $f^{n+1}\left(U_{x}\right) \subset V$. Without loss of generality, assume $U_{x} \subset G$. It is clear that $f^{n+1}\left(U_{x}\right)$ has a nonempty interior as $f$ which is semiopen. So, there exists an opene set $\widetilde{U} \subset f^{n+1}\left(U_{x}\right)$ and $N_{f}(\widetilde{U}, \mathscr{U}) \neq \varnothing$ which gives that there are $y_{1}, y_{2} \in \widetilde{U}$ and $m \in N_{f}(\widetilde{U}, \mathcal{U})$ such that

$$
\left(y_{1}, y_{2}\right) \notin \cup\left\{f^{-m}(U) \times f^{-m}(U): U \in \mathcal{U}\right\} .
$$

Let $x_{1}, x_{2} \in U_{x}$ be such that $y_{1}=f^{n+1}\left(x_{1}\right)$ and $y_{2}=f^{n+1}\left(x_{2}\right)$; then,

$$
\left(f^{n+1}\left(x_{1}\right), f^{n+1}\left(x_{2}\right)\right) \notin \cup\left\{f^{-m}(U) \times f^{-m}(U): U \in \mathcal{U}\right\} .
$$

So,

$$
\left(x_{1}, x_{2}\right) \notin \cup\left\{f^{-m-n-1}(U) \times f^{-m-n-1}(U): U \in \mathcal{U}\right\},
$$

which implies that $m+n+1 \in N_{f}(G, \mathcal{U})$. That is a contradiction.

The following theorem reveals the relations between the topological sensitivity and the classical sensitivity and generalizes the result of Theorem 2.3 of [15]. At first, we recall that $\tau_{d}$ denotes the topology of $X$ generated by the metric $d$ of $X$ if $(X, d)$ is a metric space. Let $\mathscr{F}$ be a family of $\mathbb{N}_{0}$. Review that a continuous self-map $f$ on a metric space $(X, d)$ is called $\mathscr{F}$-sensitivity if there exists $\delta>0$ such that, for each opene set $U \subset X$,

$$
\begin{aligned}
S_{f}(U, \delta)= & \left\{n \in \mathbb{N}_{0}: \exists x, y \in U \text { such that } d\left(f^{n}(x), f^{n}(y)\right)\right. \\
& >\delta\} \in \mathscr{F} .
\end{aligned}
$$


Theorem 5. Let $f$ be a continuous self-map on a metric space $(X, d)$ and let us consider the following conditions:

(1) $f:(X, d) \longrightarrow(X, d)$ is $\mathscr{F}$-sensitive

(2) $f:\left(X, \tau_{d}\right) \longrightarrow\left(X, \tau_{d}\right)$ is $\mathscr{F}$-topologically sensitive

Then, (1) implies (2). In addition, if $X$ is compact, then (2) is equivalent to (1).

Proof. The proof of Theorem 5 is similar to that of Theorem 2.3 in [15], so we omit it.

For proving the next theorem, we need firstly the following lemmas whose proofs are similar to those of the corresponding results of compact systems, but for the completeness, we include them in the paper.

Lemma 1. Assume that $\mathscr{F}$ is a proper and translation invariant family of $\mathbb{N}_{0}$. Then, a dynamical system $(X, f)$ is $\mathscr{F}$-transitive if and only if it is transitive and $\mathscr{F}$-central.

Proof. Since $\mathscr{F}$ is translation invariant, $\mathscr{F} \subset \mathscr{F}_{\text {inf }}$. If $(X, f)$ is $\mathscr{F}$-transitive, then it is transitive. So, $(X, f)$ is $\mathscr{F}$-central.

Conversely, since $(X, f)$ is transitive, for any opene subsets $U, V$ of $X$, there exists $i \in \mathbb{N}_{0}$ such that $W=U \cap f^{-i}(V) \neq \varnothing$. It is easy to prove that $N(U, V) \supseteq N(W, W)+i$. Noting that $(X, f)$ is $\mathscr{F}$-central and $\mathscr{F}$ is translation invariant, we have $N(U, V) \in \mathscr{F}$, so $(X, f)$ is $\mathscr{F}$-transitive.

Lemma 2. A dynamical system $(X, f)$ is topologically ergodic if it is topologically transitive and the set of positive upper Banach density recurrent points of $f$ is dense in $X$.

Proof. Assume that $V$ is an opene subset of $X$, by the given hypothesis, $V \cap B D^{*}(f) \neq \varnothing$. Choose $x \in V \cap B D^{*}(f)$; then, $N(x, V)$ has positive upper Banach density. Take $n_{1}, n_{2} \in N(x, V)$ with $n_{1}<n_{2}$; then, $f^{n_{1}}(x) \in V, f^{n_{2}}(x) \in V$. Set $y=f^{n_{1}}(x) \in V$; then, $x \in f^{-n_{1}}(\{y\})$. Thus,

$$
f^{n_{2}-n_{1}}(V) \cap V \supset f^{n_{2}-n_{1}}(\{y\}) \cap V \supset\left\{f^{n_{2}}(x)\right\} \cap V \neq \varnothing .
$$

Namely, $\quad n_{2}-n_{1} \in N(V, V), \quad$ note $n_{2}-n_{1} \in N(x, V)-N(x, V)$ which implies that $N(x, V)-N(x, V) \subset N(V, V)$, by Proposition 1, and $N(V, V)$ is syndetic. Therefore, $(X, f)$ is $\mathscr{F}_{\mathrm{s}}$-central. Note that $\mathscr{F}_{\mathrm{s}}$ is proper and translation invariant, by Lemma 1 , $(X, f)$ is topologically ergodic.

Next, we give one of the main results of the paper as follows. Firstly, we review that a topological space $X$ is called a Uryshon space if for every pair of distinct points $x, y \in X$, and there are two opene sets $U$ and $V$ in $X$ such that $x \in U$ and $y \in V$ and $\bar{U} \cap \bar{V}=\varnothing$. For more information about Uryshon spaces, one can refer to [18].

Theorem 6. Let $(X, f)$ be a dynamical system, where $X$ is a $T_{3}$ space. If $(X, f)$ is topologically transitive but nonminimal and the set of positive upper Banach density recurrent points of $f$ is dense in $X$, then $(X, f)$ has an $\mathscr{F}_{s}$-sensitivity cover with two elements. Therefore, $(X, f)$ is $\mathscr{F}_{s}$-topologically sensitive.

Proof. Since $(X, f)$ is not minimal, take $a \in X$ such that $X-\overline{\operatorname{orb}(a)} \neq \varnothing$, pick $b \in X-\overline{\operatorname{orb}(a)}$, then there exist $U_{b} \in \mathscr{U}_{b}$ and a neighborhood $U$ of $\overline{\operatorname{orb}(a)}$ such that $\overline{U_{b}} \cap \bar{U}=\varnothing$. Let us consider the open cover $\mathscr{W}=\left\{X-\bar{U}_{b}, X-\bar{U}\right\}$ of $X$. For any opene subset $G$ of $X$, by Lemma $2, N\left(G, U_{b}\right)$ is syndetic. Suppose $M_{1} \in \mathbb{N}$ is a gap of $j_{0} \in N\left(G, U_{b}\right)$. By the continuities of $f^{i}, i=1, \ldots, M_{1}$, there exists $U_{a} \in \mathcal{U}_{a}$ such that $f^{i}\left(U_{a}\right) \subset U$ for all $i=1, \ldots, M_{1}$. By Lemma 2 again, $N\left(G, U_{a}\right)$ is syndetic. Suppose $M_{2} \in \mathbb{N}$ is a gap of $N\left(G, U_{a}\right)$. Take $N\left(G, U_{a}\right)$ there exists $x \in G$ such that $f^{j_{0}}(x) \in U_{a}$, so $f^{j_{0}+i}(x) \in U$ for all $i=1, \ldots, M_{1}$. Clearly, there exists $0 \leq i_{0} \leq M_{1}$ such that $j_{0}+i_{0} \in N\left(G, U_{b}\right)$, so there is $y \in G$ such that $f^{j_{0}+i_{0}}(y) \in U_{b}$. Note that $f^{j_{0}+i_{0}}(y) \notin X-\bar{U}_{b}, f^{j_{0}+i_{0}}(x) \notin X-\bar{U}$, since $j_{0} \in N\left(G, U_{a}\right)$, $N\left(G, U_{a}\right)+i_{0} \subset N_{f}(G, \mathscr{W})$. Since $N\left(G, U_{a}\right)$ is syndetic and $\mathscr{F}_{\mathrm{s}}$ is a translation invariant Furstenberg family, $N_{f}(G, \mathscr{W}) \in \mathscr{F}_{s}$. It follows that $\mathscr{W}$ is an $\mathscr{F}_{\text {s }}$-sensitivity cover for $(X, f)$. Therefore, $(X, f)$ is $\mathscr{F}_{s}$-topologically sensitive.

Remark 1. Theorem 6 generalizes Theorem 2.5 of [15] to a great extent since in [17] and there exists an example showing that the set $A(f)$ of almost periodic points of $f$ may properly be contained in $B D^{*}(f)$.

In order to prove the next result, we firstly prove two lemmas whose proofs are same as those of the corresponding results of compact systems, but for the completeness of the paper, we present their complete proofs here.

Lemma 3. Let $(X, f)$ be a dynamical system. Then, $(X, f)$ is weakly mixing if and only if the family $[\mathscr{F}]$ is a filter of $\mathbb{N}_{0}$, where $\mathscr{F}=\{N(U, V): U, V$ are opene subsets of $X\}$.

Proof. If the family $[\mathscr{F}]$ is a filter, then for any opene subsets $U_{1}, U_{2}, V_{1}, V_{2}$ of $X$,

$$
N\left(U_{1} \times U_{2}, V_{1} \times V_{2}\right)=N\left(U_{1}, V_{1}\right) \cap N\left(U_{2}, V_{2}\right) \in[\mathscr{F}] .
$$

Especially, $\quad N\left(U_{1} \times U_{2}, V_{1} \times V_{2}\right) \neq \varnothing$. So, $(X, f)$ is weakly mixing.

Conversely, suppose $(X, f)$ is weakly mixing and $N\left(U_{1}, V_{1}\right), N\left(U_{2}, V_{2}\right) \in[\mathscr{F}]$. By the definition of weak mixing, there exists some $m \in N\left(U_{1}, U_{2}\right) \cap N\left(V_{1}, V_{2}\right)$. Set $A=U_{1} \cap f^{-m}\left(U_{2}\right), B=V_{1} \cap f^{-m}\left(V_{2}\right)$. For any $k \in N(A, B)$, we have

$$
\begin{aligned}
A \cap f^{-k}(B) & =U_{1} \cap f^{-m}\left(U_{2}\right) \cap f^{-k}\left(V_{1} \cap f^{-m}\left(V_{2}\right)\right) \\
& =U_{1} \cap f^{-k}\left(V_{1}\right) \cap f^{-m}\left(U_{2} \cap f^{-k}\left(V_{2}\right)\right) .
\end{aligned}
$$

This means $U_{1} \cap f^{-k}\left(V_{1}\right) \neq \varnothing$ and $U_{2} \cap f^{-k}\left(V_{2}\right) \neq \varnothing$. Then, $N(A, B) \subset N\left(U_{1}, V_{1}\right) \cap N\left(U_{2}, V_{2}\right)$. So, [F] is a filter of $\mathbb{N}_{0}$. 
Lemma 4. Let $(X, f)$ be a dynamical system. If $(X, f)$ is weakly mixing, then for each pair of opene subsets $U, V$ of $X$, $N(U, V) \in \mathscr{F}_{t}$.

Proof. Suppose $U, V$ are opene subsets of $X$. By Lemma 3, for each $n \in \mathbb{N}$,

$$
N(U, V) \cap N\left(U, f^{-1}(V)\right) \cap \cdots \cap N\left(U, f^{-n}(V)\right) \neq \varnothing .
$$

So, $N(U, V)$ is thick.

Theorem 7. Suppose that $(X, f)$ is a weakly mixing and topologically ergodic dynamical system, where $X$ admits two opene subsets $U$ and $V$ such that $\bar{U} \cap \bar{V}=\varnothing$. Then, $(X, f)$ has an $\mathscr{F}_{p s}$-sensitivity cover with two elements. Therefore, $(X, f)$ is $\mathscr{F}_{p s}$-topologically sensitive.

Proof. Obviously $\mathscr{W}=\{X-\bar{U}, X-\bar{V}\}$ is an open cover of $X$. Since $(X, f)$ is weakly mixing, by Lemma $3,[\mathscr{F}]$ is a filter of $\mathbb{N}_{0}$, where $\mathscr{F}=\{N(P, Q): P, Q$ are opene subsets of $X\}$ and each element of $[\mathscr{F}]$ is thick. Suppose that $G$ is an opene subset of $X$, then there exist a pair of opene subsets $A, B$ of $X$ such that $N(A, B) \subset N(G, U) \cap N(G, V)$ is thick. Since $(X, f)$ is topologically ergodic, $N(A, B)$ is syndetic which implies that $N(A, B)$ is piecewise syndetic. Take arbitrarily $n \in N(A, B)$ and note that $N(A, B) \subset N(G, U) \cap N(G, V)$, then $G \cap f^{-n}(U) \neq \varnothing$ and $G \cap f^{-n}(V) \neq \varnothing$. So, there exists $x, y \in G$ such that $f^{n}(x) \in U$ and $f^{n}(y) \in V$ which yields that $f^{n}(x) \notin X-\bar{U}$ and $f^{n}(y) \notin X-\bar{V}$. Therefore, $\mathscr{W}$ is an $\mathscr{F}_{\text {ps }}$-sensitivity cover for $(X, f)$ which drives that $(X, f)$ is $\mathscr{F}_{\text {ps }}$-topologically sensitive.

\section{N-Topological Sensitivity of Dynamical Systems}

In this section, we introduce the notion of $n$-sensitivity for a dynamical system. One can see [19] for the same conception for compact systems and see [20] for the difference between $n$-sensitivity and $(n+1)$-sensitivity of compact systems.

Definition 6. Let $(X, f)$ be a dynamical system and $\mathscr{U}$ be an open cover of $X$ and $n \in \mathbb{N}$. $\mathscr{U}$ is called an $n$-topological sensitivity cover for $(X, f)$ if for every opene subset $G$ of $X$, there exist $m \in \mathbb{N}_{0}$ and $n$ different points $x_{1}, x_{2}, \ldots, x_{n} \in G$ such that, for all $x, y \in\left\{x_{1}, x_{2}, \ldots, x_{n}\right\}$ with $x \neq y$,

$$
(x, y) \notin \cup\left\{f^{-m}(U) \times f^{-m}(U): U \in \mathscr{U}\right\} .
$$

Definition 7. A dynamical system $(X, f)$ (or simply the map $f)$ is called $n$-topologically sensitive if $(X, f)$ has an $n$-topological sensitivity cover.

Definition 8. Let $(X, f)$ be a dynamical system, $\mathscr{F}$ be a family of $\mathbb{N}_{0}$, and $\mathcal{U}$ be an open cover of $X . \mathscr{U}$ is called an $n$ - $\mathscr{F}$-sensitivity cover for $(X, f)$ if for each opene subset $G$ of $X$,

$$
\begin{aligned}
N_{f}^{n}(G, \mathcal{U}) & :=\left\{m \in \mathbb{N}_{0}: \exists x_{1}, x_{2}, \ldots, x_{n} \in G \text { s.t. }\left(x_{i}, x_{j}\right)\right. \\
& \left.\notin \cup\left\{f^{-m}(U) \times f^{-m}(U): U \in \mathcal{U}\right\}, i \neq j\right\} \in \mathscr{F} .
\end{aligned}
$$

Now, by using the notion of $n$ - $\mathscr{F}$-sensitivity cover, we introduce the topological version of $n$-sensitivity with respect to a Furstenberg family.

Definition 9. Let $\mathscr{F}$ be a family of $\mathbb{N}_{0}$. A dynamical system $(X, f)$ is called $n-\mathscr{F}$-topologically sensitive if $(X, f)$ admits an $n$ - $\mathscr{F}$-sensitivity cover.

In the following, we present some basic properties of $n$-topological sensitivity of dynamical systems.

Theorem 8. Let $(X, f)$ and $(Y, g)$ be two dynamical systems and $n \in \mathbb{N}$ :

(1) If $(X, f)$ and $(Y, g)$ are semiconjugate and the semiconjugation $\varphi$ from $f$ to $g$ is semi-open and $(Y, g)$ is n-topologically sensitive, then so is $(X, f)$

(2) $(X, f)$ is $n$-topologically sensitive if and only if $\left(X, f^{m}\right)$ is n-topologically sensitive for each $m \in \mathbb{N}$ if and only if $\left(X, f^{m}\right)$ is n-topologically sensitive for some $m \in \mathbb{N}$

Proof

(1) The proof is similar to that of (1) of Theorem 1, so we omit it

(2) If $\mathcal{U}$ is an $n$-topological sensitivity cover for $\left(X, f^{m}\right)$ for some $m \in \mathbb{N}$, then $\mathscr{U}$ is also an $n$-topological sensitivity cover for $(X, f)$

Now, assume that $f$ is $n$-topologically sensitive and take an $n$-topological sensitivity cover $\mathcal{U}$ for $(X, f)$. Give arbitrarily $m \in \mathbb{N}$. For every $k \in\{0, \ldots, m-1\}$, let $\mathscr{U}_{k}=\left\{f^{-k}(U): U \in \mathscr{U}\right\}$. Set $\mathscr{V}$ as the intersection of all the covers $\mathscr{U}_{0}, \ldots, \mathscr{U}_{m-1}$, i.e., $\mathscr{V}$ is the open cover of $X$ consisting of all (nonempty) sets with the form $U_{0} \cap U_{1} \cap \cdots \cap U_{m-1}$ with $U_{k} \in \mathscr{U}_{k}$ for $k=0,1, \ldots, m-1$. We claim that $\mathscr{V}$ is an $n$-topological sensitivity cover for $\left(X, f^{m}\right)$. In fact, let $G$ be an opene subset of $X$, then there exist $s \in \mathbb{N}_{0}$ and $n$ different points $x_{1}, x_{2}, \ldots, x_{n}$ in $G$ such that, for any $x_{i}, x_{j} \in\left\{x_{1}, x_{2}, \ldots, x_{n}\right\}$ with $x_{i} \neq x_{j}$,

$$
\left(x_{i}, x_{j}\right) \notin \cup\left\{f^{-s}(U) \times f^{-s}(U): U \in \mathscr{U}\right\} .
$$

Let $q \in \mathbb{N}_{0}$ and $0 \leq p<m$ such that $s=q m+p$. Now, for any $x_{i}, x_{j} \in\left\{x_{1}, x_{2}, \ldots, x_{n}\right\}$ with $x_{i} \neq x_{j}$,

$$
\left(x_{i}, x_{j}\right) \notin \cup\left\{f^{-q m-p}(U) \times f^{-q m-p}(U): U \in \mathscr{U}\right\},
$$

which implies that

$$
\left(x_{i}, x_{j}\right) \notin \cup\left\{\left(f^{m}\right)^{-q}(V) \times\left(f^{m}\right)^{-q}(V): V \in \mathscr{V}\right\} .
$$

So, $\mathscr{V}$ is an $n$-topological sensitivity cover for $\left(X, f^{m}\right)$ and $\left(X, f^{m}\right)$ is $n$-topologically sensitive. 
If $\left(X, f^{m}\right)$ is $n$-topologically sensitive for each $m \in \mathbb{N}$, then it is obvious that $\left(X, f^{m}\right)$ is $n$-topologically sensitive for some $m \in \mathbb{N}$.

About $n$-topological sensitivity, we also have the following result that is similar to Theorem 5 .

Theorem 9. Let $f$ be a continuous self-map on a metric space $(X, d)$, and let us consider the following conditions:

(1) $f:(X, d) \longrightarrow(X, d)$ is $n$-sensitive

(2) $f:\left(X, \tau_{d}\right) \longrightarrow\left(X, \tau_{d}\right)$ is n-topologically sensitive

Then, (1) implies (2). In addition, if $X$ is compact, then (2) is equivalent to (1).

Proof. The proof is similar to that of Theorem 5, so we omit it.

Theorem 10. Assume that $(X, f)$ is a weakly mixing $d y$ namical system, where $X$ is an infinite Uryshon space and there is no isolated points in $X$. Then, $(X, f)$ is n-thickly topologically sensitive for each $n \in \mathbb{N}$.

Proof. Let $n \in \mathbb{N}$. By the given conditions, there exist $n$ different points $x_{1}, x_{2}, \ldots, x_{n} \in X$ such that, for each $i=1,2, \ldots, n, x_{i}$ admits a neighborhood $U_{x_{i}}$ satisfying $\bar{U}_{x_{i}} \cap \bar{U}_{x_{j}}=\varnothing$ for all $i, j \in\{1,2, \ldots, n\}$ with $i \neq j$. Let $A=\{1, \ldots, n\}$ and

$$
\mathscr{W}=\left\{X-\bigcup_{i \in A\{1\}} \bar{U}_{x_{i}}, X-\bigcup_{i \in A\{2\}} \bar{U}_{x_{i}}, \ldots, X-\bigcup_{i \in A\{n\}} \bar{U}_{x_{i}}\right\} .
$$

We claim that $\mathscr{W}$ is an $n$-thickly topological sensitivity cover for $(X, f)$. In fact, for any opene subset $G$ of $X$, since $(X, f)$ is weakly mixing, from Lemmas 3 and 4 , it follows that

$$
H=N\left(G, U_{x_{1}}\right) \cap N\left(G, U_{x_{2}}\right) \cap \cdots \cap N\left(G, U_{x_{n}}\right) \in \mathscr{F}_{t} .
$$

For each $m \in H$, there exist $n$ distinct points $y_{1}, y_{2}, \ldots, y_{n} \in G$ such that $f^{m}\left(y_{i}\right) \in U_{x_{i}}$ for each $i \in A$; then, for any $x, y \in\left\{y_{1}, y_{2}, \ldots, y_{n}\right\}$ with $x \neq y$,

$$
(x, y) \notin \cup\left\{f^{-m}(W) \times f^{-m}(W): W \in \mathscr{W}\right\} .
$$

It follows that $(X, f)$ is $n$-thickly topologically sensitive.

Remark 2

(1) Theorem 10 generalizes Proposition 2.7 of [15] to a great extent.

(2) Fix a family $\mathscr{F}$ of $\mathbb{N}_{0}$, one can introduce the notion of $n$ - $\mathscr{F}$-topological sensitivity for dynamical systems by imitating the definition of $\mathscr{F}$-topological sensitivity. For instance, fix a family $\mathscr{F}$ of $\mathbb{N}_{0}$ and $n \in \mathbb{N}$; a dynamical system $(X, f)$ is called $n-\mathscr{F}$-topologically sensitive if there exists an open cover $\mathscr{U}$ of $X$ such that, for each opene $G \subset X$,

$$
\begin{aligned}
N_{f}^{n}(G, \mathcal{U}): & =\left\{m \in \mathbb{N}_{0}: \exists x_{1}, x_{2}, \ldots, x_{n} \in G \text { s.t. }\left(x_{i}, x_{j}\right)\right. \\
& \left.\notin \cup\left\{f^{-m}(U) \times f^{-m}(U): U \in \mathscr{U}\right\}, i \neq j\right\} \in \mathscr{F} .
\end{aligned}
$$

Then, one can obtain the similar results for $n$-F्F-topological sensitivity as Theorems $8-10$.

\section{Multitopological Sensitivity of Dynamical Systems}

In this section, we introduce the notion of multisensitivity for dynamical systems. Review that a compact system $(X, f)$ is multisensitive if there exists $\delta>0$ such that, for any finitely many opene subsets $G_{1}, \ldots, G_{l}$ of $X, \cap_{i=1}^{l} S_{f}\left(G_{i}, \delta\right) \neq \varnothing$ (see [2] for more details of multisensitivity of compact systems). One can refer to $[10,12,21]$ for the recent developments of multisensitivity for compact systems and see $[22,23]$ for the new results of multisensitivity for nonautonomous systems. Now, motivated by the notion of multisensitivity of compact systems, we introduce the conception of multisensitivity for dynamical systems as follows.

Definition 10. Let $(X, f)$ be a dynamical system. An open cover $\mathcal{U}$ of $X$ is called a multisensitivity cover for $(X, f)$ if for any finitely many opene subsets $G_{1}, \ldots, G_{n}$ of $X$, $\cap_{i=1}^{n} N_{f}\left(G_{i}, \mathscr{U}\right) \neq \varnothing$.

Definition 11. A dynamical system $(X, f)$ (or simply the map $f)$ is called multisensitive if $(X, f)$ has a multisensitivity cover.

In the following, we present some basic features of multisensitivity of dynamical systems.

Theorem 11. Let $(X, f)$ and $(Y, g)$ be two dynamical systems:

(1) If $(X, f)$ and $(Y, g)$ are semiconjugate and the semiconjugation $\varphi$ from $f$ to $g$ is semiopen and $(Y, g)$ is multisensitive, then so is $(X, f)$

(2) $(X, f)$ is multisensitive if and only if $\left(X, f^{n}\right)$ is multisensitive for each $n \in \mathbb{N}$ if and only if $\left(X, f^{n}\right)$ is multisensitive for some $n \in \mathbb{N}$

Proof

(1) The proof is similar to that of (1) of Theorem 1, so we omit it

(2) If $\mathcal{U}$ is a multisensitivity cover for $\left(X, f^{n}\right)$ for some $n \in \mathbb{N}$, then it is clear that $\mathscr{U}$ is also a multisensitivity cover for $(X, f)$

Now, assume that $f$ is multisensitive and take a multisensitivity cover $\mathscr{U}$ for $(X, f)$. Give arbitrarily $n \in \mathbb{N}$. For every $k \in\{0, \ldots, n-1\}$, let $\mathscr{U}_{k}=\left\{f^{-k}(U): U \in \mathscr{U}\right\}$. Set $\mathscr{V}$ as the intersection of all the covers $\mathscr{U}_{0}, \cdots \mathscr{U}_{n-1}$, i.e., $\mathscr{V}$ is the open cover of $X$ consisting of all (nonempty) sets with the form of $U_{0} \cap U_{1} \cap \cdots \cap U_{n-1}$ with $U_{k} \in \mathscr{U}_{k}$ for 
$k=0,1, \ldots, n-1$. We claim that $\mathscr{V}$ is a multisensitivity cover for $\left(X, f^{n}\right)$. In fact let $G_{1}, \ldots, G_{s}$ be any finitely many opene subsets of $X$, by the multisensitivity of $(X, f)$, there exist $x_{i}, \widetilde{x}_{i} \in G_{i}$ and $m \in \mathbb{N}_{0}$ such that

$$
\left(x_{i}, \tilde{x}_{i}\right) \notin \cup\left\{f^{-m}(U) \times f^{-m}(U): U \in \mathcal{U}\right\},
$$

for each $1 \leq i \leq s$. Let $q \in \mathbb{N}_{0}$ and $0 \leq p<n$ such that $m=q n+p$. Now, we have for all $1 \leq i \leq s$,

$$
\begin{aligned}
\left(x_{i}, \tilde{x}_{i}\right) & \notin \cup\left\{f^{-q n-p}(U) \times f^{-q n-p}(U): U \in \mathcal{U}\right\} \\
& =\cup\left\{\left(f^{n}\right)^{-q}\left(f^{-p}\right)(U) \times\left(f^{n}\right)^{-q}\left(f^{-p}\right)(U): U \in \mathcal{U}\right\},
\end{aligned}
$$

which implies

$$
\left(x_{i}, \widetilde{x}_{i}\right) \notin \cup\left\{\left(f^{n}\right)^{-q}(V) \times\left(f^{n}\right)^{-q}(V): V \in \mathscr{V}\right\},
$$

for each $1 \leq i \leq s$. So, $q \in N_{f^{n}}\left(G_{i}, \mathscr{V}\right)$ for every $1 \leq i \leq s$, namely, $\cap_{i=1}^{s} N_{f^{n}}\left(G_{i}, \mathscr{V}\right) \neq \varnothing$. Therefore, $\mathscr{V}$ is a multisensitivity cover for $\left(X, f^{n}\right)$ and $\left(X, f^{n}\right)$ is multisensitive.

If $\left(X, f^{n}\right)$ is multisensitive for each $n \in \mathbb{N}$, clearly $\left(X, f^{n}\right)$ is multisensitive for some $n \in \mathbb{N}$. (2).

Summarize the above proof process, and we prove result

About multisensitivity, we have the following Theorem 12 that is similar to Theorems 5 and 9.

Theorem 12. Let $f$ be a continuous self-map on a metric space $(X, d)$, and let us consider the following conditions:

(1) $f:(X, d) \longrightarrow(X, d)$ is multisensitive

(2) $f:\left(X, \tau_{d}\right) \longrightarrow\left(X, \tau_{d}\right)$ is multisensitive

Then, (1) implies (2). In addition, if $X$ is compact, then (2) is equivalent to (1).

Proof. The proof is similar to that of Theorem 9, so we omit it.

Theorem 13. Suppose that $(X, f)$ is a weakly mixing $d y$ namical system and $X$ admits two opene subsets $U$ and $V$ of $X$ such that $\bar{U} \cap \bar{V}=\varnothing$. Then, $(X, f)$ is multisensitive.

Proof. By the conditions there exist two opene sets $U, V \subset X$ such that $\bar{U} \cap \bar{V}=\varnothing$. Set $\mathscr{W}=\{X-\bar{U}, X-\bar{V}\}$. Then, $\mathscr{W}$ is a multisensitivity cover of $(X, f)$. In fact, for any finitely many opene sets $U_{1}, \ldots, U_{n}$ of $X$, since $(X, f)$ is weakly mixing, by Lemma $3, N\left(U_{i}, U\right)$ and $N\left(U_{i}, V\right)$ are thick for each $1 \leq i \leq n$ and $A:=\cap_{i=1}^{n}\left(N\left(U_{i}, U\right) \cap N\left(U_{i}, V\right)\right) \neq$ $\varnothing$. Then, for each $k \in A$ and each $1 \leq i \leq n$, there exist $x_{i}, y_{i} \in U_{i}$ such that $f^{k}\left(x_{i}\right) \in U, f^{k}\left(y_{i}\right) \in V$ which yields that $f^{k}\left(x_{i}\right) \notin X-\bar{U}$ and $f^{k}\left(y_{i}\right) \notin X-\bar{V}$. So, $A \subset N_{f}\left(U_{i}, \mathscr{W}\right) \neq \varnothing$ for all $1 \leq i \leq n$. It follows that $\cap_{i=1}^{n} N_{f}\left(U_{i}, \mathscr{W}\right) \neq \varnothing$. Therefore, $\mathscr{W}$ is a multisensitivity cover for $(X, f)$ and $(X, f)$ is multisensitive.

Theorem 14. Let $(X, f)$ be an $M$-system satisfying; there exist two minimal sets $A, B \subset X$ and two open sets $U, V \subset X$ such that $A \subset U, B \subset V$, and $\bar{U} \cap \bar{V}=\varnothing$, then $(X, f)$ is $\mathscr{F}_{\text {ts }}$-topologically sensitive.

Proof. We directly prove that $\mathscr{W}=\{X-\bar{U}, X-\bar{V}\}$ is an $\mathscr{F}_{t s}$-sensitivity cover for $(X, f)$. Let $W$ be any opene subset of $X$. For each $k \in \mathbb{N}$, since $f, f^{2}, \ldots, f^{k}$ are continuous and $A$ is $f$-invariant, $f^{-i}(U)$ is an open set containing $A$ for all $1 \leq i \leq k$. By the assumption of $(X, f)$ being an $M$-system, there is a minimal point $z_{i} \in f^{-i}(U)-A$ such that $\overline{\operatorname{orb}\left(z_{i}\right)} \cap A=\varnothing$ for each $1 \leq i \leq k$. Let $U_{A}^{i}=f^{-i}(U)-\overline{\operatorname{orb}\left(z_{i}\right)}$, $1 \leq i \leq k$, then $U_{A}=\cap_{i=1}^{k} U_{A}^{i}$ is an open set containing $A$. So, $A \subset U_{A} \subset f^{-i}(U)$ for each $1 \leq i \leq k$. Since $(X, f)$ is transitive, $N\left(W, U_{A}\right) \neq \varnothing$. Hence, there are $x \in W$ and $m \in \mathbb{N}$ such that $f^{m}(x) \in f^{m}(W) \cap U_{A}$. By the continuity of $f^{m}$, there is an open set $W_{x}$ such that $x \in W_{x} \subset W$ and $f^{m}\left(W_{x}\right) \subset U_{A}$. So, there exists a minimal point $\tilde{x} \in W_{x}$ of $f$ such that $f^{m}(\tilde{x}) \in U_{A}$. Note that $f^{m}(\tilde{x})$ is also a minimal point of $f$, then there exists a syndetic set $\left\{n_{j}\right\}$ of $\mathbb{N}_{0}$ such that $f^{n_{j}+m}(\tilde{x}) \in U_{A}^{i}$ for all $j \geq 1$ and each $1 \leq i \leq k$, so $f^{n_{j}+m+i}(\widetilde{x}) \in f^{i}\left(U_{A}^{i}\right) \subset U$ for each $1 \leq i \leq k$. This shows that $N(W, U)$ is a thickly syndetic set. By the same argument, $N(W, V)$ is also thickly syndetic. Thus, $N(W, U) \cap N(W, V)$ is thickly syndetic, which implies that $\mathscr{W}$ is an $\mathscr{F}_{\text {ts }}$-sensitivity cover for $(X, f)$ and $(X, f)$ is $\mathscr{F}_{t s}$-topologically sensitive.

Lemma 5. If $(X, f)$ is multisensitive and there is no isolated points in $X$, then $\cap_{i=1}^{n} N_{f}\left(U_{i}, \mathcal{U}\right)$ is infinite for any finitely many opene sets $U_{1}, \ldots, U_{n}$ of $X$, where $\mathcal{U}$ is a multisensitivity cover for $(X, f)$.

Proof. In fact, if there exist finitely many opene sets $G_{1}, \ldots, G_{k}$ of $X$ such that $\cap_{i=1}^{k} N_{f}\left(G_{i}, \mathcal{U}\right)$ is finite, put

$$
m=\max \left\{n: n \in \bigcap_{i=1}^{k} N_{f}\left(G_{i}, \mathcal{U}\right)\right\} \text {, }
$$

then $m \in N_{f}\left(G_{i}, \mathcal{U}\right)$ for all $i=1, \ldots, k$. Thus, there exist $x_{i}, y_{i} \in G_{i}$ such that

$$
\left(x_{i}, y_{i}\right) \notin \cup\left\{f^{-m}(U) \times f^{-m}(U): U \in \mathcal{U}\right\},
$$

for every $1 \leq i \leq k$. Note that, for every $1 \leq j \leq m$, there exists $W_{i, j} \in \mathcal{U}$ such that $f^{j}\left(x_{i}\right) \in W_{i, j}$. By the continuities of $f^{j}$, $1 \leq j \leq m$, there is an open neighbourhood $U_{i, j} \subset G_{i}$ of $x_{i}$ such that $f^{j}\left(U_{i, j}\right) \subset W_{i, j}$ for each $1 \leq j \leq m$ and $1 \leq i \leq k$. Now, for $1 \leq i \leq k$, let $\widetilde{G}_{i}=\cap_{j=1}^{m} U_{i, j} \subset G_{i}$, and it is easy to check that $f^{j}\left(\widetilde{G}_{i}\right) \subset W_{i, j} \in \mathcal{U}$ for every $1 \leq j \leq m$ and $1 \leq i \leq k$. Thus, $\left.\cap_{i=1}^{k} N_{f}\left(\widetilde{G}_{i}, \mathcal{U}\right)\right\}>m$ and $\cap_{i=1}^{k} N_{f}\left(G_{i}, \mathcal{U}\right)>m$. That is a contradiction.

Theorem 15. Let $(X, f)$ be a dynamical system, where $f$ is a surjection. If $(X, f)$ is multisensitive, then $(X, f)$ is thickly topologically sensitive. Moreover, if $(X, f)$ is point-transitive, then the converse holds.

Proof. Firstly assume that $(X, f)$ is multisensitive with a multisensitivity cover $\mathcal{U}$ of $X$ and $k \in \mathbb{N}$. For any opene set $G \subset X$, we choose opene sets $G_{i} \subset f^{-i}(G)$ for each $i=1, \ldots, k$. By Lemma $5, \cap_{i=1}^{k} N_{f}\left(G_{i}, \mathcal{U}\right)$ is infinite, so we 
can choose some $n_{k} \in \cap_{i=1}^{k} N_{f}\left(G_{i}, \mathscr{U}\right)$ with $n_{k}>k$. Note that $n_{k} \in N_{f}\left(G_{i}, \mathcal{U}\right)$ for each $i=1, \ldots, k$, and there exist $x_{i}, y_{i} \in G_{i}$ which gives that $f^{n_{k}}\left(x_{i}\right), f^{n_{k}}\left(y_{i}\right) \in f^{n_{k}-i}(G)$ such that

$$
\left(x_{i}, y_{i}\right) \notin \cup\left\{f^{-n_{k}}(U) \times f^{-n_{k}}(U): U \in \mathcal{U}\right\} .
$$

Then, for each $i=1, \ldots, k$, there exist $\tilde{x}_{i}, \widetilde{y}_{i} \in G$ such that $f^{n_{k}-i}\left(\tilde{x}_{i}\right)=f^{n_{k}}\left(x_{i}\right), f^{n_{k}-i}\left(\tilde{y}_{i}\right)=f^{n_{k}}\left(y_{i}\right)$, and

$$
\left(\widetilde{x}_{i}, \widetilde{y}_{i}\right) \notin \cup\left\{f^{-\left(n_{k}-i\right)}(U) \times f^{-\left(n_{k}-i\right)}(U): U \in \mathcal{U}\right\},
$$

which implies $n_{k}-i \in N_{f}(G, \mathcal{U})$ for all $i=1, \ldots, k$. Therefore, $(X, f)$ is thickly topologically sensitive.

Now, we assume $(X, f)$ is thickly topologically sensitive with a thickly topological sensitivity cover $\mathcal{U}$ of $X$ and $x$ is a transitive point of $(X, f)$. Let $k \in \mathbb{N}$ and $U_{1}, \ldots, U_{k}$ be opene subsets of $X$. Then, for each $i=1, \ldots, k$, there exists $n_{i} \in \mathbb{N}$ such that $f^{n_{i}}(x) \in U_{i}$. Hence, there exists $V \in \mathcal{U}_{x}$ such that $f^{n_{i}}(V) \in U_{i}$ for each $i=1, \ldots, k$. By the given assumptions, there is $s \in \mathbb{N}_{0}$ such that

$$
\left\{s, s+1, \ldots, s+n_{1}+n_{2}+\cdots+n_{k}\right\} \subset N_{f}(V, \mathcal{U}) .
$$

So, $s+n_{i} \in N_{f}(V, \mathcal{U})$ for all $i=1, \ldots, k$ which yields that there exist $x_{i}, y_{i} \in V$ such that

$$
\left(x_{i}, y_{i}\right) \notin \cup\left\{f^{-\left(s+n_{i}\right)}(U) \times f^{-\left(s+n_{i}\right)}(U): U \in \mathcal{U}\right\},
$$

for all $i=1, \ldots, k$. Therefore, $f^{n_{i}}\left(x_{i}\right), f^{n_{i}}\left(y_{i}\right) \in f^{n_{i}}(V) \subset U_{i}$ for each $i=1, \ldots, k$ and

$$
\left(f^{n_{i}}\left(x_{i}\right), f^{n_{i}}\left(y_{i}\right)\right) \notin \cup\left\{f^{-s}(U) \times f^{-s}(U): U \in \mathcal{U}\right\},
$$

which implies that $s \in N_{f}\left(U_{i}, \mathcal{U}\right)$ for every $i=1, \ldots, k$, i.e., $s \in \cap_{i=1}^{k} N_{f}\left(U_{i}, \mathcal{U}\right)$. Therefore, $(X, f)$ is multisensitive.

Remark 3. For the related studies of thickly topological sensitivity and multisensitivity of semigroups, one can refer to [24] in which all kinds of sensitivities are defined via a uniform structure of the involved space. In this paper, we introduce such sensitivities by an open cover of the phase space, so they are a little different.

\section{Data Availability}

No data are used to support this study.

\section{Conflicts of Interest}

The authors declare that there are no conflicts of interest regarding the publication of this paper.

\section{Acknowledgments}

This work was supported by the National Natural Science Foundation of China (11261039 and 11661054).

\section{References}

[1] D. Ruelle, "Dynamical systems with turbulent behavior," in Mathematical Problems in Theoretical Physics, vol. 80, pp. 341-360, Springer, Berlin, Germany, 1978.
[2] T. K. S. Moothathu, "Stronger forms of sensitivity for dynamical systems," Nonlinearity, vol. 20, no. 9, pp. 2115-2126, 2007.

[3] R. Devaney, "An introduction to chaotic dynamical systems," Ddison-Weeley Studies in Nonlinearitypp. 224-231, AddisonWesley Publishing Company Advanced Book Program, Redwood City, CA, USA, 2nd edition, 1989.

[4] J. Banks, J. Brooks, G. Cairns, G. Davis, and P. Stacey, “On Devaney's definition of chaos," The American Mathematical Monthly, vol. 99, no. 4, pp. 332-324, 1992.

[5] B. Frédérick and M. Étienne, "Dynamics of linear operators," Cambridge Tracts in Mathematics, vol. 179, Cambridge University Press, Cambridge, UK, 2009.

[6] K.-G. Grosse-Erdmann and P. Alfred, Linear Chaos, SpringerVerlag, London, UK, 2011.

[7] K. Marko, Chaos for Linear Operators and Abstract Differential Equations, Nova Science Publishers, New York, NY, USA, 2020.

[8] F. Tan and R. Zhang, "On $\mathscr{F}$-sensitive pairs," Acta Mathematica Scientia, vol. 31, no. 4, pp. 1425-1435, 2011.

[9] G. Zhang, "Relativization of complexity and sensitivity," Ergodic Theory and Dynamical Systems, vol. 27, no. 4, pp. 1349-1371, 2007.

[10] W. Huang, S. Kolyada, and G. Zhang, "Analogues of Auslander-Yorke theorems for multi-sensitivity," Ergodic Theory and Dynamical Systems, vol. 38, no. 2, pp. 651-665, 2018.

[11] R. Li, "A note on stronger forms of sensitivity for dynamical systems," Chaos, Solitons \& Fractals, vol. 45, no. 6, pp. 753-758, 2012.

[12] V. Radhika and D. Ruchi, "On stronger forms of sensitivity in non-autonomous systems," Taiwanese Journal of Mathematics, vol. 22, no. 5, pp. 1139-1159, 2018.

[13] J. Yin and Z. Zhou, "Weakly almost periodic points and some chaotic properties of dynamical systems," International Journal of Bifurcation and Chaos, vol. 25, no. 9, Article ID 1550115, 2015.

[14] W. Huang, D. Khilko, S. Kolyada, and G. Zhang, "Dynamical compactness and sensitivity," Journal of Differential Equations, vol. 260, no. 9, pp. 6800-6827, 2016.

[15] A. Fedeli, "Topologically sensitive dynamical systems," Topology and Its Applications, vol. 248, pp. 192-203, 2018.

[16] H. Furstenberg, Recurrence in Ergodic Theory and Combinational Number Theory, Princeton University Press, Princeton, NJ, USA, 1981.

[17] X. Y. Wang and Y. Huang, "Recurrence of transitive points in dynamical systems with the specification property," Acta Mathematica Sinica, English Series, vol. 34, no. 12, pp. 1879-1891, 2018.

[18] S. Willard, General Topology, Addison-Wesley, Princeton, NJ, USA, 1970.

[19] J. Xiong, "The chaos in transitive dynamical systems," Science China Mathematics, vol. 35, no. 3, pp. 302-311, 2005.

[20] S. Shao, X. Ye, and R. Zhang, "Sensitivity and regionally proximal relation in minimal systems," Science in China Series A: Mathematics, vol. 51, no. 6, pp. 987-994, 2008.

[21] P. Oprocha, T. Yu, and G. Zhang, "Multi-sensitivity, multitransitivity and $\Delta$-transitivity. Dynamics: topology and numbers," Contemporary Mathematics, vol. 744, pp. 231-244, 2020.

[22] S. Mohammad and D. Ruchi, "Furstenberg family and multisensitivity in non-autonomous systems," Journal of Difference Equations and Applications, vol. 25, no. 12, pp. 1755-1767, 2019. 
[23] S. Mohammad and D. Ruchi, "Multi-sensitivity and other stronger forms of sensitivity in non-autonomous discrete systems," Chaos Solitons Fractal, vol. 115, pp. 341-348, 2018.

[24] H. Wang, "Thickly sydetic sensitivity of semi-groups," Bulletin of the Korean Mathematical Society, vol. 55, no. 4, pp. 1125-1135, 2018. 